\title{
Salud auto-referida y desigualdades sociales, ciudad de Buenos Aires, Argentina, 2005
}

\author{
Self-rated health and social inequalities, \\ Buenos Aires, Argentina, 2005
}

\footnotetext{
${ }^{1}$ Maestría en Epidemiología Gestión y Políticas de Salud, Universidad Nacional de Lanús, Lanús, Argentina. 2 Center for Social Epidemiology and Population Health, University of Michigan, Ann Arbor, U.S.A.

Correspondencia M. Alazraqui

Maestría en Epidemiología, Gestión y Políticas de Salud, Universidad Nacional de Lanús.

Maza 1966, Ciudad

Autónoma de Buenos Aires

C1240ADP, Argentina.

malazraqui@yahoo.com.ar
}

\section{Abstract}

Self-rated health is a quality-of-life indicator. This study investigates the impact of individual-level and neighborhood-level socioeconomic characteristics, considered simultaneously, on the state of self-rated health at the individual level in Buenos Aires, Argentina. The study employs a two-level (individual and neighborhood) multilevel analysis, and the data sources were the 2005 Argentina National Risk Factor Survey (multistage probabilistic sample) and the 2001 Population Census. Linear regression shows that higher schooling and income, as well as occupational category, are related to better self-rated health, and increasing age with worse health. In the multilevel analysis, an increase in the proportion (per census tract) of individuals with less schooling was associated with an increase in the proportion of individuals with worse self-rated health. Improving the general health of the population requires strategies and action that reduce the levels of social inequalities in their multiple dimensions, including the individual and neighborhood levels.

Diagnosis of Health Situation; Quality of Life; Social Inequity
Marcio Alazraqui 1

Ana V. Diez Roux 2

Nancy Fleischer 2

Hugo Spinelli 1

\section{Introducción}

La salud auto-referida (auto-percepción de la salud) es un indicador de calidad de vida que concierne a la percepción de la propia salud por parte de los individuos consultados. Es utilizada cada vez en mayor magnitud en estudios epidemiológicos, debido a su validez en predecir la morbi-mortalidad y el decremento funcional en personas mayores; así como en el seguimiento de grupos poblacionales con problemas de salud específicos y en la medición de la efectividad de las intervenciones implementadas 1,2 .

Diferentes instrumentos se encuentran disponibles para determinar la salud auto-referida, como el cuestionario de calidad de vida SF-36 (http://www.sf-36.org), el cuestionario Euroqol5D (http://www.euroqol.org) y una escala visual numérica, semejante a un termómetro de calidad de vida (medida en grados de 0 a 100) 3 . El SF-36, utilizado en esta investigación, tiene una escala tipo Likert de fácil aplicación en la población adulta.

La salud auto-referida presenta variantes de acuerdo al sexo, edad, raza, etnia, niveles de educación, ingresos per capita, situación laboral, entre otros factores a nivel individual 4,5,6,7.

La percepción que tiene cada individuo de su salud está relacionada, además, con sus grupos de pertenencia y el contexto en que vive 8 , y por eso la importancia de comprender cómo se interrelacionan los niveles individual y grupal 
en la auto-referencia de salud individual. Para incorporar los niveles de análisis mencionados se utilizan diseños que sean capaces de describir el patrón de salud auto-referida, a nivel individual, como el resultado simultáneo de características a nivel individual y características a nivel grupal. Este tipo de estudio se denomina diseño multinivel, que tiene la potencialidad de integrar variables independientes provenientes de diferentes niveles de análisis en un modelo jerárquico ${ }^{9}$.

Se encuentran relativamente pocos estudios de producción latinoamericana que utilicen un abordaje que considere la variación de la salud auto-referida a nivel individual como resultado de aspectos individuales y grupales considerados simultáneamente 10 .

La presente investigación se desarrolla en la ciudad de Buenos Aires, que forma parte del mayor conglomerado urbano de la Republica Argentina. La población en el Censo Nacional de Población, Hogares y Viviendas 2001 era 2.776.138 habitantes (hombres 1.258.458 y mujeres 1.517.680) 11 , con una alta densidad poblacional en zona urbana $\left(13,679.6\right.$ habitantes $\left./ \mathrm{km}^{2}\right) 12$ y gran heterogeneidad de condiciones socioeconómicas 13 .

La presente investigación aborda la siguiente cuestión: ¿cuál es el impacto de las características demográficas y socioeconómicas a nivel individual y de las características socioeconómicas de vecindad en el estado de salud auto-referida a nivel individual?

\section{Material y métodos}

Se utilizó el diseño de análisis multinivel que permite estudiar de forma simultánea los efectos de variables individuales y variables grupales en los resultados individuales 9,14. El modelo multinivel utilizado en esta investigación está constituido por dos niveles: un primer nivel individual (personas entrevistadas) y un segundo nivel grupal (vecindad del entrevistado); el resultado correspondió a una única variable dependiente a nivel individual: el estado de salud auto-referido.

Las fuentes de información utilizadas fueron la Encuesta Nacional de Factores de Riesgo realizada en 2005, y el Censo Nacional de Población, Hogares y Viviendas realizado en 2001; ambos fueron obtenidos en la Dirección General de Estadística y Censos del Gobierno de la Ciudad de Buenos Aires. La información del nivel individual (primer nivel del modelo) se obtuvo de la Encuesta Nacional de Factores de Riesgo; mientras que la información del nivel grupal (segundo nivel del modelo) se obtuvo del Censo Nacional de Población, Hogares y Viviendas.
Los datos de la Encuesta Nacional de Factores de Riesgo fueron obtenidos, tanto en formato magnético, como en los cuestionarios elaborados en papel 15. Estos últimos fueron utilizados para agregar la fracción censal, correspondiente a la dirección de residencia de los entrevistados, a la base de datos original, a fin de relacionar sus características individuales con las características de su vecindad. En la base de datos original no figuraba la fracción censal del domicilio del entrevistado, porque no era el objetivo expreso de la Encuesta Nacional de Factores de Riesgo y por eso fue necesaria la realización de la carga de datos mencionada. El procedimiento de adjudicación del domicilio al área geográfica de referencia (fracción censal) se realizó a través de la georreferencia, según información de los planos de la ciudad contenida en el software georreferencial utilizado (MapInfo Professional 7; MapInfo Corp., New York, Estados Unidos). El procedimiento de georreferencia consiste en adjudicar un punto del plano (coordenadas geográficas del domicilio del entrevistado) a un polígono del plano (fracción censal donde se ubica el domicilio del entrevistado). Se georreferenció la totalidad de los domicilios de los entrevistados según la fracción censal de residencia, ya que la muestra utilizada tenía la totalidad de los domicilios de los individuos entrevistados 16 .

La población sujeta al muestreo por la Encuesta Nacional de Factores de Riesgo fueron los residentes en viviendas particulares de la ciudad de Buenos Aires mayores de 17 años. Se realizó un muestreo probabilístico multietápico, que consistió en la selección de personas a través de 4 etapas: las dos primeras correspondieron al marco de muestreo nacional urbano 16 , cuyo universo comprende a viviendas particulares en aglomeraciones de al menos 5.000 habitantes 17 . A partir del proceso mencionado quedaron seleccionadas áreas donde la Encuesta Nacional de Factores de Riesgo realizó un muestreo sistemático para la selección de viviendas, donde identificó hogares y en cada hogar seleccionó al azar un individuo mayor de 17 años al que aplicó la encuesta 18.

Los datos fueron recolectados por entrevistadores capacitados por el Instituto Nacional de Estadística y Censos (INDEC), que realizaron entrevistas personales en los domicilios de las personas seleccionadas en la muestra. La encuesta estaba compuesta de un cuestionario del hogar 19 y un cuestionario individual 20. El primero contenía preguntas sobre características demográficas y socioeconómicas del hogar; y el segundo sobre características demográficas, socioeconómicas y de condiciones de salud del entrevistado. Los cuestionarios utilizados tomaron como modelo 
instrumentos internacionales que fueron validados localmente a través de una adaptación transcultural 21 .

La variable dependiente a nivel individual fue la salud general auto-referida del entrevistado que presentaba las siguientes opciones: mala, regular, buena, muy buena y excelente (escala de peor a mejor). Esta variable mostró una alta correlación con la variable estado de salud, que correspondía a la salud auto-referida, medida a través de una escala visual numérica en grados.

Las principales variables independientes, y sus valores posibles, utilizados a nivel individual (primer nivel del modelo) en esta investigación fueron los siguientes: sexo (masculino, femenino); edad (en años); educación (analfabeto hasta primaria completa, secundaria incompleta, terciaria o universitaria incompleta, terciaria o universitaria completa); ingresos mensuales del hogar (\$0-\$600, \$601-\$1.000, \$1.001-\$1.500, \$1.501-\$2.000, \$2.001 o más, no sabe/no contesta); y situación laboral (desocupado, inactivo, asalariado, cuenta propia, patrón).

Todas las variables a nivel individual fueron utilizadas según las definiciones de la Encuesta Nacional de Factores de Riesgo 3, a excepción de la situación laboral, donde se combinaron las variables: condición de actividad y categoría ocupacional.

La principal variable independiente utilizada a nivel grupal (segundo nivel del modelo) fue la proporción de educación secundaria incompleta o inferior, medida a nivel de la fracción censal de residencia del entrevistado a partir del Censo Nacional de Población, Hogares y Viviendas realizado en 2001. Se trata de una variable grupal derivada 9. La fracción censal urbana es una división territorial que contiene entre 3.000 y 5.000 viviendas 22 , y fue considerada para los fines de esta investigación como una variable proxy de vecindad (características sociodemográficas de la vecindad del entrevistado).

El análisis de los datos se realizó utilizando modelos de regresión lineal y modelos lineales de análisis multinivel. Se utilizaron modelos lineales por su simpleza y porque la variable dependiente tenía una distribución aproximadamente normal.

Los modelos de regresión lineal fueron definidos de la siguiente forma, según sus variables componentes:

- Modelo 1: salud general y educación (referencia utilizada: educación terciaria o universitaria completas), ajustadas por sexo y edad;

- Modelo 2: salud general e ingresos (referencia utilizada: mayor ingreso de \$2.000), ajustados por sexo y edad;
- Modelo 3: salud general y situación laboral (referencia utilizada: asalariados), ajustadas por sexo y edad;

- Modelo 4: salud general, educación, ingresos y situación laboral, ajustados por sexo y edad. Se utilizaron las mismas referencias mencionadas en los modelos descriptos anteriormente. Una variante del Modelo 4 consideró a hombres y mujeres por separado.

En todos los modelos la referencia utilizada en la variable sexo fueron las mujeres. Se calculó la significancia estadística ( $\mathrm{p}<0.05$ ) para cada uno de los valores de las variables, y la $\mathrm{p}$ de tendencia. La p de tendencia se obtuvo a través de la inclusión de las categorías de la variable en cuestión como variable ordinal. En la variable ingreso se excluyeron los ingresos ignorados en el cálculo de la p de tendencia.

Los modelos utilizados en el análisis multinivel fueron definidos de la siguiente forma según sus variables componentes.

- Modelo 1: nivel 1, salud general, sexo y edad; y nivel 2, proporción de educación secundaria incompleta o inferior;

- Modelo 2: nivel 1, salud general, sexo, edad, educación, ingresos y situación laboral; y nivel 2, proporción de educación secundaria incompleta o inferior.

Todos los modelos multinivel incluyeron un intercepto aleatorio para cada fracción censal, a fin de tener en cuenta la correlación entre individuos pertenecientes al mismo vecindario. En los modelos se incluyeron las variables educación e ingresos mensuales del hogar como variables continuas.

Se realizó una exploración de los modelos utilizados con y sin pesos, no encontrándose diferencias relevantes y se optó por presentar resultados sin pesos por considerarlos de más fácil interpretación.

Los softwares utilizados en el trabajo fueron Epi Info 6 para la carga de datos (Centers for Disease Control and Prevention, Atlanta, Estados Unidos), MapInfo Professional 7, Stat Transfer 5 para conversión de archivos (Circle Systems, Seatle, Estados Unidos), Intercooled Stata 7 para análisis de datos (Stata Corp., College Station, Estados Unidos) y HLM 5 para análisis multinivel (Scientific Software International, Lincolnwood, Estados Unidos).

Los cuidados éticos consistieron en la protección de la confidencialidad de las personas entrevistadas, tanto en las bases de datos como en los resultados publicados, según la Ley Nacional no. 17.622 y sus decretos reglamentarios en relación al secreto estadístico. 


\section{Resultados}

La Encuesta Nacional de Factores de Riesgo entrevistó en la ciudad de Buenos Aires a 1.510 individuos. Presentó un porcentaje de no respuesta de los hogares de $18,4 \%$, y un porcentaje de no respuesta individual de $2,9 \% 23$.

Los entrevistados en su mayoría fueron mujeres (59,3\%); y la media de edad fue mayor en este grupo (49,6 años en mujeres y 45,4 años en varones). Las mujeres presentaron menor nivel de educación; un 52,7\% de las mujeres tenían hasta secundaria completa (inclusive), en comparación con el 45,6\% de los hombres. Las mujeres entrevistadas presentaron menor ingreso mensual del hogar, con un $45,5 \%$ hasta $\$ 1.000$; mientras los hombres entrevistados tuvieron el $33,2 \%$ en ese nivel de ingresos. La situación laboral presentó en condición de inactividad $37,2 \%$ en mujeres y $17,7 \%$ en hombres, y por ende los porcentuales en las otras categorías de situación laboral fueron menores en mujeres (Tabla 1).

Tabla 1

Características demográficas, sociales, de salud y del área de residencia. Ciudad de Buenos Aires, Argentina, 2005.

\begin{tabular}{|c|c|c|}
\hline Variable & Hombre $(\mathrm{N}=615)$ & Mujer $(\mathrm{N}=895)$ \\
\hline Edad (años) [Media \pm DS (rango)] & $45,4 \pm 18,9(18-97)$ & $49,6 \pm 19,9(18-95)$ \\
\hline \multicolumn{3}{|l|}{ Educación [n (\%)] } \\
\hline Hasta primaria completa & $92(14,9)$ & $184(20,5)$ \\
\hline Secundaria incompleta & $84(13,6)$ & $102(11,4)$ \\
\hline Secundaria completa & $105(17,0)$ & $186(20,7)$ \\
\hline Terciaria o universitaria incompleta & $163(26,5)$ & $175(19,5)$ \\
\hline Terciaria o universitaria completa & $171(27,8)$ & $248(27,7)$ \\
\hline \multicolumn{3}{|l|}{ Ingreso mensual del hogar (pesos) [n (\%)] } \\
\hline No sabe/No contesta & $56(9,1)$ & $112(12,5)$ \\
\hline $0-600$ & $86(13,9)$ & $205(22,9)$ \\
\hline $601-1.000$ & $119(19,3)$ & $203(22,6)$ \\
\hline $1.001-1.500$ & $107(17,4)$ & $125(13,9)$ \\
\hline $1.501-2.000$ & $99(16,1)$ & $115(12,8)$ \\
\hline $2.001+$ & $148(24,0)$ & $135(15,0)$ \\
\hline \multicolumn{3}{|l|}{ Situación laboral [n (\%)] } \\
\hline Desocupado & $28(4,5)$ & $30(3,3)$ \\
\hline Inactivo & $109(17,7)$ & $333(37,2)$ \\
\hline Asalariado & $309(50,2)$ & $373(41,6)$ \\
\hline Cuenta propia & $123(20,0)$ & $134(14,9)$ \\
\hline Patrón & $46(7,4)$ & $25(2,7)$ \\
\hline \multicolumn{3}{|l|}{ Salud general [n (\%)] } \\
\hline Mala & $12(1,9)$ & $24(2,6)$ \\
\hline Regular & $67(10,8)$ & $125(13,9)$ \\
\hline Buena & $239(38,8)$ & $366(40,8)$ \\
\hline Muy buena & $213(34,6)$ & $270(30,1)$ \\
\hline Excelente & $84(13,6)$ & $110(12,2)$ \\
\hline \multicolumn{3}{|l|}{ Estado de salud } \\
\hline Media \pm SD (rango) & $77,6 \pm 15,6(0-100)$ & $74,7 \pm 17,9(0-100)$ \\
\hline Mediana & 80 & 80 \\
\hline Percentilos 25-75 & $70-90$ & $70-90$ \\
\hline \multicolumn{3}{|c|}{ Porcentaje de educación secundaria incompleta o } \\
\hline \multicolumn{3}{|l|}{ inferior en fracción censal } \\
\hline Media \pm DS (rango) & $37,9 \pm 13,0(17,3-79,1)$ & $38,3 \pm 13,5(17,3-79,1)$ \\
\hline Mediana & 36,0 & 36,4 \\
\hline Percentilos 25-75 & $29,7-46,0$ & $29,7-46,2$ \\
\hline
\end{tabular}

Fuente: elaboración propia a partir de la Encuesta Nacional de Factores de Riesgo de la Ciudad de Buenos Aires 2005 y Censo Nacional de Población, Hogares y Viviendas realizado en 2001; ambos obtenidos en la Dirección de Estadística y Censos del Gobierno de la Ciudad de Buenos Aires. 
El 16,5\% de las mujeres y al 12,7\% de los hombres refirieron tener salud mala o regular. El estado de salud medido en una escala en grados (de 0 a 100) fue peor en las mujeres en relación a los hombres; 77,6 \pm 15,6 (0-100) en hombres y 74,7 \pm 17,9 (0-100) (Tabla 1), en mujeres. Se exploró la relación entre las variables salud general y estado de salud y se encontró una muy alta correlación entre ambas tanto en el total, como en hombres y mujeres por separado. En relación a estas dos últimas variables, se decidió utilizar la salud general en los modelos, en lugar de estado de salud, luego de realizar un análisis descriptivo y encontrar una buena correlación entre las mismas (coeficiente de correlación de Spearman 0,6274). Por ello, el resto de los análisis se limitaron a una sola de estas variables: la salud general.

En la muestra se obtuvo una media de 9,74 $( \pm 4,33)$ individuos por fracción censal (con el correspondiente desvío estándar); con una mediana de 9,00 individuos por fracción censal. El percentil 25 correspondió a 7 individuos y el percentil 75 a 10 individuos por fracción censal.

Las características socioeconómicas de las fracciones censales en que residían los entrevistados fueron semejantes a las de fracciones censales en donde no residían entrevistados. La comparación se realizó a partir de las siguientes variables: proporción de mujeres, proporción sin cobertura de salud, proporción con educación secundaria incompleta o inferior, proporción con educación terciaria o superior, proporción con necesidades básicas insatisfechas, proporción de viviendas con materiales adecuados, proporción de viviendas con 2 o más personas por cuarto, proporción de viviendas con 3 o más personas por cuarto. No se encontraron diferencias estadísticamente significativas utilizada la prueba $t$ (valor p) en las variables de la fracciones censales incluidas o no en la muestra de entrevistados.

En el Modelo 1 de regresión lineal observamos que a medida que disminuye el nivel de educación se observa un descenso gradual en el nivel de salud general; y esta tendencia fue estadísticamente significativa ( $\mathrm{p}$ de tendencia $<0,0001$ ) (Tabla 2).

En el Modelo 2, a medida que disminuye en nivel de ingresos mensuales del hogar, se observa un descenso gradual en el nivel de salud general; y esta tendencia es lineal y estadísticamente significativa ( $p$ de tendencia $<0,0001$ ) (Tabla 2). Se realizó una exploración con la categoría de ingresos ignorados y debido a su propia naturaleza se ha comportado de forma heterogénea en los modelos, por lo que no fue presentado.

En el Modelo 3 las situaciones laborales correspondientes a desocupado e inactivo se relacionaron con disminución de la salud general; y los cuenta-propistas (autónomos) y patrones con un aumento de la misma. Solamente la categoría de inactivos fue estadísticamente significativa (Tabla 2).

En el Modelo 4, en que se encuentran todas las variables mencionadas, observamos las mismas tendencias expuestas en los tres modelos anteriores con algunas variaciones menores, y menos categorías estadísticamente significativas. La p de tendencia fue estadísticamente significativa, tanto para educación e ingresos tomados de forma asilada, como para ambos en el modelo completo (Tabla 2). Esto indica que tanto educación como ingreso se asocian independientemente con el nivel de salud general.

En relación al sexo, la salud general auto-referida era peor en mujeres, aunque los valores del coeficiente son muy cercanos a 0 en los cuatro modelos, y además no son estadísticamente significativos. A medida que se incrementa la edad en todos los modelos es menor el nivel de salud general, y además es estadísticamente significativo (Tabla 2).

El modelo de regresión lineal que considera diferencias medias de forma separada, asociadas a salud general en hombres y mujeres, ajustadas por edad, educación, ingresos y situación laboral tiene resultados muy semejantes al modelo anterior. En general, se observan tendencias semejantes en las variables consideradas en el modelo anterior, aunque algunas no son tan marcadas; y presentan menos valores estadísticamente significativos. La p de tendencia es estadísticamente significativa en las variables edad, educación e ingresos (Tabla 3).

Se realizaron pruebas estadísticas para verificar la existencia de interacción entre la variable sexo y las variables educación e ingresos y las mismas no fueron estadísticamente significativas.

Los modelos multinivel se utilizaron para estimar diferencias medias en salud general asociadas a características del vecindario (el porcentaje de residentes con educación secundaria incompleta o inferior), después de ajustar por sexo y edad, y luego de ajustar por sexo, edad, educación, ingresos, y situación laboral. En el Modelo 1 multinivel, a medida que la proporción de educación secundaria incompleta o inferior de la fracción censal aumenta, se produce un decremento de la salud general a nivel individual, y es estadísticamente significativo. El Modelo 2 multinivel muestra que la proporción de educación secundaria incompleta o inferior del vecindario continúa significativamente asociada a la salud general, tras ajustar por características socioeconómicas a nivel individual: por cada unidad que aumenta el porcentaje de educación 
Diferencias medias ajustadas en salud general (intervalo de confianza del 95\% entre paréntesis), asociadas a características individuales. Ciudad de Buenos Aires, Argentina, 2005

\begin{tabular}{|c|c|c|c|c|}
\hline Variables & Modelo 1 & Modelo 2 & Modelo 3 & Modelo 4 \\
\hline \multicolumn{5}{|l|}{ Sexo } \\
\hline Mujer (referencia) & - & - & - & - \\
\hline Hombre & $-0,028(-0,117 ; 0,059)$ & $-0,001(-0,091 ; 0,088)$ & $-0,009(-0,101 ; 0,082)$ & $0,009(-0,080 ; 0,100)$ \\
\hline Edad & $-0,013(-0,016 ;-0,011)$ * & $-0,016(-0,018 ;-0,014)$ * & $-0,016(-0,019 ;-0,013)$ * & $-0,012(-0,015 ;-0,009)$ * \\
\hline \multicolumn{5}{|l|}{ Educación } \\
\hline Universitaria completa (referencia) & - & - & - & - \\
\hline Universitaria incompleta & $-0,039(-0,166 ; 0,087)$ & - & - & $0,015(-0,114 ; 0,145)$ \\
\hline Secundaria completa & $-0,175(-0,303 ;-0,046)$ * & - & - & $-0,114(-0,246 ; 0,018)$ \\
\hline Secundaria incompleta & $-0,265(-0,413 ;-0,118)$ * & - & - & $-0,169(-0,324 ;-0,015)$ * \\
\hline Primaria completa & $-0,598(-0,735 ;-0,462)$ * & - & - & $-0,467(-0,617 ;-0,318)$ * \\
\hline$p$ de tendencia & 0,000 & - & - & 0,000 \\
\hline \multicolumn{5}{|l|}{ Ingresos (pesos) } \\
\hline $2.001+$ (referencia) & - & - & - & - \\
\hline $1.501-2.000$ & - & $-0,090(-0,243 ; 0,062)$ & - & $-0,049(-0,202 ; 0,102)$ \\
\hline $1.001-1.500$ & - & $-0,111(-0,261 ; 0,038)$ & - & $-0,018(-0,170 ; 0,133)$ \\
\hline $601-1.000$ & - & $-0,303(-0,442 ;-0,164)$ * & - & $-0,177(-0,321 ;-0,034)$ * \\
\hline $0-600$ & - & $-0,461(-0,607 ;-0,315)$ * & - & $-0,244(-0,403 ;-0,085)$ * \\
\hline p de tendencia ** & - & 0,000 & - & 0,001 \\
\hline \multicolumn{5}{|l|}{ Situación laboral } \\
\hline Desocupado & - & - & $-0,187(-0,421 ; 0,046)$ & $-0,093(-0,324 ; 0,136)$ \\
\hline Inactivo & - & - & $-0,167(-0,289 ;-0,045)$ * & $-0,106(-0,229 ; 0,016)$ \\
\hline Asalariado (referencia) & - & - & - & - \\
\hline Cuenta propia & - & - & $0,037(-0,089 ; 0,164)$ & $-0,000(-0,124 ; 0,124)$ \\
\hline Patrón & - & - & $0,159(-0,055 ; 0,374)$ & $0,053(-0,157 ; 0,265)$ \\
\hline
\end{tabular}

Fuente: elaboración propia a partir de la Encuesta Nacional de Factores de Riesgo de la Ciudad de Buenos Aires 2005 y Censo Nacional de Población, Hogares y Viviendas realizado en 2001; ambos obtenidos en la Dirección de Estadística y Censos del Gobierno de la Ciudad de Buenos Aires.

Nota: los modelos utilizados en la regresión lineal fueron definidos de la siguiente forma: Modelo 1 (salud general y educación, ajustados por sexo y edad); Modelo 2 (salud general e ingresos, ajustados por sexo y edad); Modelo 3 (salud general y situación laboral, ajustados por sexo y edad); Modelo 4 (salud general, educación, ingresos y situación laboral, ajustados por sexo y edad). Los valores de referencia utilizados se encuentran señalados en cada variable.

* Resultados estadísticamente significativos $(p<0,05)$;

** En la variable ingreso se excluyeron los ingresos ignorados en el cálculo del p de tendencia.

secundaria incompleta o inferior la salud general disminuye en promedio 0.362 puntos. Todas las demás variables incorporadas de forma conjunta se comportan de manera semejante a los modelos anteriormente descriptos. El mayor nivel de educación (estadísticamente significativo), el mayor nivel de ingresos, el sexo masculino y las ocupaciones cuenta-propista (autónomos) y patrón se relacionan con un incremento en la salud general; así como la edad (estadísticamente significativo), el sexo femenino y las condiciones de desocupado e inactivo se relacionan con un decremento en la salud general a nivel individual (Tabla 4).

Los coeficientes de correlación intraclase fueron en el Modelo 1 multinivel 0,01236 y en el
Modelo 2 multinivel 0,00792. Los coeficientes de correlación intraclase indican el grado de semejanza entre unidades del nivel inferior, pertenecientes a la misma unidad o agrupación de nivel superior. En este caso, la correlación intraclase mide el grado en que la salud general es semejante para individuos pertenecientes a un mismo vecindario 9 .

\section{Discusión}

En este estudio los hombres presentaron en promedio mayor nivel de educación formal, mayores ingresos y mejor situación laboral que las mujeres. La salud auto-referida fue mejor en 
Diferencias medias ajustadas en salud general (intervalo de confianza del 95\% entre paréntesis) asociadas a características individuales para varones y mujeres.

\begin{tabular}{|c|c|c|}
\hline \multirow[t]{2}{*}{ Variables } & \multicolumn{2}{|c|}{ Modelo 4} \\
\hline & Hombres & Mujeres \\
\hline Edad & $-0,013(-0,017 ;-0,008)$ * & $-0,012(-0,015 ;-0,008)$ * \\
\hline \multicolumn{3}{|l|}{ Educación } \\
\hline Universitaria completa (referencia) & - & - \\
\hline Universitaria incompleta & $0,060(-0,136 ; 0,256)$ & $-0,036(-0,212 ; 0,140)$ \\
\hline Secundaria completa & $-0,033(-0,247 ; 0,181)$ & $-0,182(-0,353 ;-0,011)$ * \\
\hline Secundaria incompleta & $-0,182(-0,413 ; 0,492)$ & $-0,168(-0.378 ; 0,041)$ \\
\hline Primaria completa & $-0,362(-0,606 ;-0,118)$ * & $-0,550(-0.742 ;-0,358)$ * \\
\hline $\mathrm{p}$ de tendencia & 0,002 & 0,000 \\
\hline \multicolumn{3}{|l|}{ Ingresos (pesos) } \\
\hline $2.001+$ (referencia) & - & - \\
\hline $1.501-2.000$ & $0,003(-0,216 ;-0,223)$ & $-0,127(-0,343 ; 0,088)$ \\
\hline $1.001-1.500$ & $0,073(-0,147 ; 0,294)$ & $-0,126(-0,339 ; 0,087)$ \\
\hline $601-1.000$ & $-0,175(-0,396 ; 0,044)$ & $-0,201(-0,395 ;-0,006)$ * \\
\hline $0-600$ & $-0,327(-0,584 ;-0,071)$ * & $-0,236(-0,445 ;-0,027)$ * \\
\hline $\mathrm{p}$ de tendencia** & 0,018 & 0,027 \\
\hline \multicolumn{3}{|l|}{ Situación laboral } \\
\hline Desocupado & $-0,238(-0,570 ; 0,093)$ & $0,067(-0,254 ; 0,390)$ \\
\hline nactivo & $-0,124(-0,341 ; 0,091)$ & $-0,100(-0,252 ; 0,051)$ \\
\hline Asalariado (referencia) & - & - \\
\hline Cuenta propia & $0,075(-0,105 ; 0,255)$ & $-0,076(-0,249 ; 0,097)$ \\
\hline Patrón & $0,022(-0,242 ; 0,288)$ & $0,100(-0,251 ; 0,452)$ \\
\hline
\end{tabular}

Fuente: elaboración propia a partir de la Encuesta Nacional de Factores de Riesgo de la Ciudad de Buenos Aires 2005 y Censo Nacional de Población, Hogares y Viviendas realizado en 2001; ambos obtenidos en la Dirección de Estadística y Censos del Gobierno de la Ciudad de Buenos Aires.

Nota: el modelo utilizado en la regresión lineal fue definido de la siguiente forma: Modelo 4 (salud general, educación ingresos y situación laboral, ajustados por edad). Los valores de referencia utilizados se encuentran señalados en cada variable.

* Resultados estadísticamente significativos $(p<0,05)$;

** En la variable ingreso se excluyeron los ingresos ignorados en el cálculo del p de tendencia.

los hombres también, tanto medida en una escala tipo Likert (salud general), como a través de una escala visual numérica en grados (estado de salud).

Los modelos de regresión lineal presentados indican que el mayor nivel de educación, el mayor nivel de ingresos y una mayor categoría en la situación laboral considerados por separado están relacionados con una mejor salud autoreferida. El aumento de la edad está relacionado en los modelos con peor salud auto-referida. El modelo completo, que incluye simultáneamente las variables mencionadas, muestra resultados semejantes a los modelos anteriores, inclusive cuando se analiza hombres y mujeres por separado.

El modelo multinivel indica la importancia de la variable grupal, proporción de educación secundaria incompleta o inferior en la fracción censal en el resultado de la salud auto-referida a nivel individual. Es decir, a medida que el proporción de educación secundaria incompleta o inferior aumenta (nivel grupal) se produce un decremento de la salud general auto-referida del entrevistado (nivel individual). Las otras variables incorporadas al modelo de forma conjunta se comportan de manera semejante a los modelos anteriormente descriptos. En su conjunto los resultados indican que hay importantes diferencias en salud general, asociadas a desigualdades socioeconómicas a nivel individual, y al contexto socioeconómico del vecindario.

La Encuesta Nacional de Factores de Riesgo encontró a nivel del país un nivel de salud autoreferida regular o malo en 19,9\% de la población (en mujeres $22,6 \%$ y en hombres $17 \%$ ); y la ciu- 
Diferencias medias en salud general asociadas a características del área de residencia, antes y después de ajustar por características socioeconómicas a nivel individual. Ciudad de Buenos Aires, Argentina, 2005

\begin{tabular}{|c|c|c|c|c|}
\hline \multirow[t]{2}{*}{ Variables } & \multicolumn{2}{|c|}{ Modelo 1} & \multicolumn{2}{|c|}{ Modelo 2} \\
\hline & Diferencia media & Valor de $\mathrm{p}$ & Diferencia media & Valor de $\mathrm{p}$ \\
\hline \multicolumn{5}{|c|}{ Proporción de educación secundaria } \\
\hline incompleta o inferior & $-0,786$ & 0,000 & $-0,362$ & 0,000 \\
\hline Sexo * & 0,039 & 0,368 & 0,005 & 0,896 \\
\hline Edad (años) & $-0,018$ & 0,000 & $-0,014$ & 0,000 \\
\hline Educación & - & - & 0,109 & 0,000 \\
\hline Ingresos & - & - & 0,021 & 0,116 \\
\hline Desocupado ** & - & - & $-0,111$ & 0,264 \\
\hline Inactivo ** & - & - & $-0,103$ & 0,063 \\
\hline Cuenta propia ** & - & - & 0,012 & 0,842 \\
\hline Patrón ** & - & - & 0,072 & 0,453 \\
\hline
\end{tabular}

Fuente: elaboración propia a partir de la Encuesta Nacional de Factores de Riesgo de la Ciudad de Buenos Aires 2005 y Censo Nacional de Población, Hogares y Viviendas realizado en 2001; ambos obtenidos en la Dirección de Estadística y

Censos del Gobierno de la Ciudad de Buenos Aires.

Nota: los modelos utilizados en el análisis multinivel fueron definidos de la siguiente forma:

Modelo 1 tiene en el nivel 1 las variables: salud general, sexo y edad; y en el nivel 2 la variable: proporción de educación secundaria incompleta o inferior.

Modelo 2 tiene en el nivel 1 las variables: salud general, sexo, edad, educación, ingresos y situación laboral; y en el nivel 2 la variable: proporción de educación secundaria incompleta o inferior. La variable situación laboral tiene como valores posibles desocupado, inactivo, asalariado (que se utilizó como referencia), cuenta propia y patrón.

* Referencia: mujeres;

** Referencia: asalariado.

dad de Buenos Aires presentó uno de los valores más bajos del país, 15,3\% (en mujeres 16,6\% y en hombres 13,8\%). En relación a los hombres, las mujeres estudiadas presentaron mayor media de edad, menor nivel de educación, menor ingreso mensual del hogar, y mayor inactividad laboral. Las mujeres presentaron una salud auto-referida peor que los hombres, a pesar de presentar en general una mayor expectativa de vida, enfermedades de mejor pronóstico y de mayor duración 24. La Encuesta Nacional de Factores de Riesgo tuvo como objetivo el estudio de los factores de riesgo para enfermedades cardiovasculares y demás características asociadas a nivel individual 15; sin tener en cuenta cómo interactúan estas características individuales en relación a características grupales.

Los resultados de esta investigación son compatibles con las escasas investigaciones en Latinoamérica sobre salud auto-referida, a partir de modelos que tienen en cuenta la interrelación entre diferentes niveles de análisis 10; así como con otras investigaciones de desigualdades sociales y sus implicancias en salud 4,7,25. En la ciudad de Buenos Aires se encontró - a partir de un análisis espacial - una asociación entre áreas (fracciones censales) con menores niveles de educación y mayores tasas de mortalidad total, cardiovascular y respiratoria. Estos hallazgos que relacionan desigualdades sociales con desigualdades en salud son congruentes con los resultados de la presente investigación 26 .

Esta investigación utiliza fuentes de datos secundarios del nivel nacional correspondientes al Ministerio de Salud y Ambiente y al INDEC del Ministerio de Economía y Producción. Estos datos oficiales fueron generados a través de una serie de procedimientos normatizados y obtenidos por personal cualificado, a fin de garantizar una calidad adecuada. Son datos de acceso público y sus resultados generales fueron publicados para distintos propósitos, aunque diferentes de los objetivos de esta investigación. Estos aspectos revelan el cuidado por la validez y acceso a los datos utilizados en esta investigación.

LaEncuesta Nacional de Factores de Riesgo en la ciudad de Buenos Aires presentó un porcentaje de no respuesta de los hogares $(18,4 \%)$, mayor que el promedio del país $(10,4 \%)$ y un porcentaje de no respuesta individual $(2,9 \%)$, menor que el 
promedio del país $(3,2 \%)$. Se considera porcentaje de no respuesta de los hogares, cuando el entrevistador no logra concertar la entrevista por diversas razones (rechazo, ausencia de sus moradores, u hogar sin mayores de 17 años), sobre el total de viviendas de la muestra; y el porcentaje de no respuesta individual cuando no se obtiene respuesta por ausencia y/o rechazo a participar de la persona seleccionada sobre el total de individuos seleccionados 18,23. Si la no respuesta se asocia simultáneamente al bajo nivel socioeconómico y a tener salud referida mala o regular, es posible que nuestros resultados subestimen la magnitud de las desigualdades en salud referida.

La salud-autoreferida es un indicador subjetivo, aunque muestra semejanzas con indicadores objetivos del estado de salud ${ }^{1}$. Se encuentran diferencias entre la salud auto-referencia por hombres y mujeres. En las mujeres la auto-referencia de un bajo nivel de salud está relacionada con peor calidad de vida, mientras en los hombres se relaciona con una alta probabilidad de muerte 27. Estas diferencias son atribuidas a que las respuestas de las mujeres son basadas en un amplio rango de factores relacionados o no con la salud en relación a los hombres 28; y por lo tanto se recomienda estudiar por separado hombres y mujeres, como se hizo en nuestro estudio.

Debido a que no fueron incluidas todas las fracciones censales de la ciudad de Buenos Aires en la muestra, una de las limitaciones potenciales de esta investigación podría ser la diferencia entre las fracciones censales incluidas o no. Se realizó una exploración a fin de evaluar la similitud entre las fracciones censales incluidas o no incluidas en la muestra y se concluyó que las fracciones censales de la muestra no diferían en gran medida de las fracciones censales no incluidas en la muestra en las variables estudiadas.

En esta investigación se concibe a la fracciones censales como un proxy de vecindad, como se ha empleado en otras investigaciones 10,26,29. Las fracciones censales son definidas por el Censo Nacional de Población, Hogares y Viviendas a partir de aspectos operativos censales, como la organización del trabajo del censista, y también tiene en cuenta criterios demográficos, económicos y socioculturales ${ }^{22}$. Estas unidades espaciales definidas con criterios censales tienen la ventaja de disponer de información sociodemográfica, aunque no conforman estrictamente una unidad espacial socio-política. Su utilización debe ser crítica, a fin de profundizar en la comprensión de cómo operan los procesos causales que articulan las características de la vecindad con las características de los individuos 8,30,31,32; y así colaborar en la implementación de intervenciones más eficaces.

Las diferencias de contexto entre las unidades espaciales estudiadas no son naturales, sino el resultado de políticas públicas específicas, o a la ausencia de las mismas. La identificación de contextos de vecindad diferentes debería orientar la definición de políticas públicas dirigidas a incrementar el nivel de salud de la población 30 .

Se exploraron diversas variables censales a nivel agregado y se optó por utilizar solamente la proporción de educación secundaria incompleta o inferior a nivel de la fracción censal de residencia del entrevistado. El nivel de educación ha sido descrito como un buen indicador de condiciones de vida 33; y la proporción de educación secundaria incompleta o inferior fue utilizada en otras investigaciones 26 , mostrándose consistente con los objetivos propuestos en la investigación. Es de tener en cuenta que la ciudad de Buenos Aires es la jurisdicción con mayor nivel educacional (terciario o universitario completo) de Argentina.

En la búsqueda de modelos de investigación que den cuenta de la complejidad de los eventos del proceso salud-enfermedad-atención-cuidado en poblaciones se encuentran los modelos multinivel 14,34. Los factores individuales no son suficientes para explicar la variabilidad geográfica de las diferencias en la salud auto-referida, ya que también influyen las características de los grupos en donde se encuentran los individuos, y en el caso de la presente investigación la zona de residencia. El modelo multinivel presentado en esta investigación aporta al conocimiento de la relación entre la salud auto-referida y el contexto grupal (vecindad) en que el individuo se encuentra. Este fue el objetivo de la presente investigación, que la diferencia de la Encuesta Nacional de Factores de Riesgo y la complementa. Este conocimiento es de utilidad en la comprensión del estado de salud de las poblaciones y un insumo relevante en la implementación de políticas públicas orientadas a incrementar el nivel de salud de la población. 


\section{Resumen}

La salud auto-referida es un indicador de calidad de vida. ¿Cuál es el impacto de las características socioeconómicas a nivel individual y a nivel de la vecindad, consideradas simultáneamente, en el estado de salud auto-referida a nivel individual? Diseño de análisis multinivel con dos niveles: individual y vecindad. Las fuentes de información fueron: Encuesta Nacional de Factores de Riesgo (muestreo probabilístico multietápico) y Censo Poblacional. La regresión lineal muestra que mayores niveles de educación, de ingresos y de categoría laboral se relacionan con mejor salud auto-referida; y el aumento de la edad con peor salud. En el análisis multinivel, a medida que la proporción de individuos con educación inferior aumentaba en la fracción censal, aumentaba también la proporción de individuos con peor salud auto-referida. Mejorar la salud general de la población requerirá estrategias y acciones que disminuyan los niveles de desigualdad social en sus múltiples dimensiones, individual y de vecindad.

Diagnóstico de la Situación en Salud; Calidad de Vida; Inequidad Social

\section{Referencias}

1. Idler EL, Benyamini Y. Self-rated health and mortality: a review of twenty-seven community studies. J Health Soc Behav 1997; 38:21-37.

2. Velarde-Jurado E, Figueroa CA. Evaluación de la calidad de vida. Salud Pública Méx 2002; 44:349-61.

3. Ministerio de Salud y Ambiente, Instituto Nacional de Estadística y Censos. Encuesta nacional de factores de riesgo 2005: documento para la utilización de la base de datos-usuario. Buenos Aires: Ministerio de Salud y Ambiente, Instituto Nacional de Estadística y Censos; 2005.

4. Dachs JNW. Determinantes das desigualdades na auto-avaliação do estado de saúde no Brasil: análise dos dados da PNAD/1998. Ciênc Saúde Coletiva 2002; 7:641-57.

5. Instituto Nacional de Câncer, Secretaria de Vigilância em Saúde, Ministério da Saúde. Tabelas do inquérito domiciliar sobre comportamentos de risco e morbidade referida de doenças e agravos não transmissíveis: Brasil, 17 capitais e Distrito Federal 2002-2005. Rio de Janeiro: Instituto Nacional de Câncer; 2005.

6. Lethbridge-Çejku M, Schiller JS, Bernadel L. Summary health statistics for U.S. adults: National Health Interview Survey, 2002. Vital Health Stat 10 2004; (222):1-151.

\section{Colaboradores}

M. Alazraqui, A. V. Diez Roux, N. Fleischer y H. Spinelli participaron en la elaboración de la idea central, del texto y en la revisión final.

\section{Agradecimientos}

Agradecemos a Guillermo Macías, Osvaldo Santiago, Débora Lev y Gabriela Zunino de la Universidad Nacional de Lanús que colaboraron en la carga de las bases de datos y en la georreferencia; así como a Martín Moreno, Victoria Mazzeo, Susana Frías y Martín Méndez de la Dirección General de Estadística y Censos del Gobierno de la Ciudad de Buenos Aires que colaboraron en la obtención de información necesaria para la investigación; y a José Tapia Granados por sus sugerencias en la revisión del artículo. Al Fogarty Institute y National Institutes of Health (application number RO3 TW007020) por el financiamiento.
7. Barata RB, Almeida MF, Montero CV, Silva ZP. Health inequalities based on ethnicity in individuals aged 15 to 64, Brazil, 1998. Cad Saúde Pública 2007; 23:305-13.

8. Diez Roux AV. Investigating neighborhood and area effects on health. Am J Public Health 2001; 91:1783-9.

9. Diez Roux AV. A glossary for multilevel analysis. J Epidemiol Community Health 2002; 56:588-94.

10. Santos SM, Chor D, Werneck GL, Coutinho ESF. Associação entre fatores contextuais e auto-avaliação de saúde: uma revisão sistemática de estudos multinível. Cad Saúde Pública 2007; 23:2533-54.

11. Instituto Nacional de Estadística y Censos. Ciudad de Buenos Aires: población por sexo según edad en años simples, año 2001. http://www.indec.gov. ar/censo2001s2_2/datos/02000c61.xls (accedido el 30/Mar/2008).

12. Instituto Nacional de Estadística y Censos. Ciudad de Buenos Aires según Distrito Escolar: población, superficie y densidad, años 1991-2001. http://www.indec.gov.ar/censo2001s2_2/datos/ 02000c22.xls (accedido el 30/Mar/2008).

13. Carello G, Gratti P, Mai V. Calidad de vida en la ciudad de Buenos Aires: una propuesta de configuración de espacios homogéneos. Población de Buenos Aires 2006; 3:7-28. 
14. Diez Roux AV. Multilevel analysis in public health research. Annu Rev Public Health 2000; 21:171-92.

15. Ministerio de Salud y Ambiente. ¿Qué es la Encuesta Nacional de Factores de Riesgo 2005? http://www.msal.gov.ar/htm/Site/enfr/conteni dos/PDF/EFR_marzo06_c_tapas.pdf (accedido el 20/Mar/2008).

16. Instituto Nacional de Estadística y Censos. Marco de muestreo nacional urbano para encuestas a hogares. Buenos Aires: Instituto Nacional de Estadística y Censos; 1999. (Metodologías INDEC, 12).

17. Messere MR, Hoszowski AE. Encuesta permanente de hogares: actualización del diseño de sus muestras, 1974-2003. http://www.indec.gov.ar/ nuevaweb/cuadros/4/eph_muestras_74-03.pdf (accedido el 19/Jul/2007).

18. Ministerio de Salud y Ambiente. Aspectos metodológicos. http://www.msal.gov.ar/htm/Site/enfr/ contenidos/PDF/02_Metodologicos.pdf (accedido el 23/Dic/2007).

19. Ministerio de Salud y Ambiente. Cuestionario del hogar. http://www.msal.gov.ar/htm/Site/enfr/ contenidos/PDF/3\%20Anexo\%20III\%20b\%20 Cuestionario\%20del\%20hogar.pdf (accedido el 23/ Dic/2007).

20. Ministerio de Salud y Ambiente. Cuestionario individual. http://www.msal.gov.ar/htm/Site/enfr/ contenidos/PDF/3\%20Anexo\%20III\%20c\%20 Cuestionario\%20individual.pdf (accedido el 23/ Dic/2007).

21. Ministerio de Salud de la Nación. Vigilancia de enfermedades no transmisibles: encuesta nacional de factores de riesgo. Boletín Epidemiológico Periódico, Edición Especial 2004. http://www.msal. gov.ar/htm/Site/enfr/contenidos/PDF/boletin especialENT.pdf (accedido el 23/Dic/2007).

22. Instituto Nacional de Estadística y Censos. Censo Nacional de Población y Viviendas 2001. http:// www.indec.gov.ar/webcenso/provincias_2/casp met.doc (accedido el 20/Mar/2008).

23. Ministerio de Salud y Ambiente. Encuesta Nacional de Factores de Riesgo: resumen de resultados principales. Provincia: Ciudad Autónoma de Buenos Aires. http://www.msal.gov.ar/htm/Site/ enfr/contenidos/PDF/INFORME-provincial/cap. pdf (accedido el 23/Dic/2007).
24. Ministerio de Salud y Ambiente. Salud general y calidad de vida. http://www.msal.gov.ar/htm/ Site/enfr/contenidos/PDF/05_SaludGeneral.pdf (accedido el 23/Dic/2007).

25. Diez Roux AV, Link BG, Northridge ME. A multilevel analysis of income inequality and cardiovascular disease risk factors. Soc Sci Med 2000; 50:673-87.

26. Diez Roux AV, Green Franklin T, Alazraqui M, Spinelli $\mathrm{H}$. Intraurban variations in adult mortality in a large Latin American city. J Urban Health 2007; 84:319-33.

27. Maia FOM, Duarte YAO, Lebrão ML, Santos JLF. Fatores de risco para mortalidade em idosos. Rev Saúde Pública 2006; 40:1049-56.

28. Benyamini Y, Leventhal EA, Leventhal H. Gender differences in processing information for making self-assessments of health. Psychosom Med 2000; 62:354-64.

29. Alazraqui M, Mota E, Spinelli H, Guevel C. Desigualdades en salud y desigualdades sociales: un abordaje epidemiológico en un municipio urbano de Argentina. Rev Panam Salud Pública 2007; 21: 1-10.

30. Diez Roux AV. Neighborhoods and health: where are we and were do we go from here? Rev Épidémiol Santé Publique 2007; 55:13-21.

31. Diez Roux AV, Javier Nieto F, Caulfield L, Tyroler HA, Watson RL, Szklo M. Neighbourhood differences in diet: the Atherosclerosis Risk in Communities (ARIC) Study. J Epidemiol Community Health 1999; 53:55-63.

32. Lisabeth LD, Diez Roux AV, Escobar JD, Smith MA, Morgenstern LB. Neighborhood environment and risk of ischemic stroke: the brain attack surveillance in Corpus Christi (BASIC) project. Am J Epidemiol 2007; 165:279-87.

33. Nunes A, Santos JRS, Barata RB, Vianna SM. Medindo as desigualdades em saúde no Brasil: uma proposta de monitoramento. Brasília: Organização Pan-Americana da Saúde/Organização Mundial da Saúde/Instituto de Pesquisa Econômica Aplicada; 2001.

34. Diez Roux AV. En defensa de una epidemiología con números [Editorial]. Salud Colect 2007; 3 : 117-9.

Recibido el 08/Nov/2008

Versión final presentada el 20/Mar/2009

Aprobado el 13/May/2009 